\title{
Fault Tolerance Mechanism for Internet of Things (IoT) by Solving Nature Inspired Computing Algorithm (NIC)
}

\author{
A. Prasanth Rao ${ }^{1}$, K. K. C. Deekshit ${ }^{2}$, Gopala Venu Madhav ${ }^{3,}$ C. Nagamani ${ }^{4}$ \\ ${ }^{1}$ Dept. of IT, Anurag University, Hyderabad, India \\ ${ }^{2}$ Dept. of EEE, Sreenidhi Institute of Science \& Technology, Hyderabad, India \\ ${ }^{3,4}$ Dept. of EEE, Anurag University, Hyderabad, India \\ Email: 'Iadirajupppy@yahoo.com
}

\begin{abstract}
In clustering approach the sensor nodes are grouped to form a cluster. The nodes of a clustering network have low powered battery capability and limited processing capabilities. These nodes continuously exchange the data to cluster head which is in turn transforming the data to its base station. Few of these nodes in network may be faulty or may not support life time processing data due to its low power battery. All these sensor nodes measure the temperature, humidity, sound and pollution from environment and collected data is send to cloud for further processing. The fault tolerance mechanism of these nodes is solved by applying genetic algorithm by implementing chromosome technique to identify and avoid fault nodes in the network. This proposed research work increases detection of fault nodes in a network, increase network efficiency, lifetime and reach energy optimization results in Internet of Things (IoT) concept. The performance evaluation shows that the data accuracy in Genetic Algorithm (GA) is higher when compared with Direct Diffusion (DD) Algorithm and Ad-hoc on demand Distance Vector (AODV) Algorithm.
\end{abstract}

Keywords: Clustering, Internet of Things (IoT), Fault tolerance mechanism, Nature Inspired Computing (NIC), Genetic Algorithm (GA)

\section{Introduction}

Clustering support network device supports data routing for Internet of Things (IoT) concept. When nodes in the cluster network become battery down or become faulty, then the rest of the nodes should perform task without any disruption in transmitting the routing scheme and faulty nodes are detected, avoided to create ultimate routing path for performing better clustering approach in Internet of Things (IoT) concept. In this paper, authors proposed Efficient and Intelligent Fault-tolerance Network and Dynamic Algorithm (EIFDA) to provide fast routing, network path diagnosis, and reconstruct the network for path for routing in IoT. EIFDA based fault-tolerance algorithm performs due to its ability of fast routing recovery mechanism and prolonging the lifetime of the IoT.
In clustering, sensor possesses manufacturing of design problems, battery energy problems, and topological problems that affect the performance level of sensor nodes in a network. While in design problems, the problems like low latency, fault tolerance, scalability and coverage problems are there. By these problems the nodes unplanned to deploy in not in controlled areas for that reason the nodes become faultynodes. For that reason the nodes will not connect and communicate with base station. Another problem is with topology issues like without proper network the communication from source to target will not get proper communication for routing.

In clustering approach having some challenges to overcome problems for communicating and routing. The first challenge is maximize Energy efficiency and minimize power consumption to enhance lifetime of sensor nodes. The life is very limit for sensor Nodes because these nodes efficiency depends on battery power. Every time battery power will be improved. The second challenge is bandwidth problem. Between two sensor nodes the data transmission for one time nearby $10-100 \mathrm{~kb} / \mathrm{s}$ bandwidth is required. Third challenge is deployment of sensor nodes, the node deployment follow static or dynamic as per specifications. The last challenging is security [1] for sensor nodes in clustering of IoT approach. The sensor node authentication and authorization are security of nodes.

Fault may occur for sensor nodes due to in-proper deployment of sensor nodes position, network errors, energy depletion, communication, etc. There are three types of faults in sensor nodes concept i.e., both in network, or hardware and also in software. In network fault occur communication and network connection failure. Hardware fault occurs due to memory and power supply. Software fault occurs when sensor nodes in-correct values collected or created and when these values send to base station. The fault diagnosis is one of method to collect fault status information of sensor nodes and enhance network status, enhance life-time of nodes and reduce network problem.

\section{Related Work}

Lilia Paradis et.al. [2] summarize and compare existing fault tolerant methods for solving fault tolerant 
problems occur in sensor nodes. Mihaela Cardeiet.al [3] proposed fc-degree analyst topology (fc-ATC) problem for control fault-tolerant sensor topology that minimizes sensor transmission power is minimized. Mihaele Cardeiet.al [4] proposed Bayesian fusion algorithm to mix data trust and communication trust components to make trust communication between nodes. This algorithm is very trusty to add and remove these components whenever needed. The trustiness is provided and managed by these components whether to know nodes are trustworthiness or untrustworthy status. Ayon Charaborty et.al. [5] proposed method of improve energy efficiency and network lifetime by applying Genetic algorithm inspired GROUP routing protocol. This algorithm enhances network performance by providing sub-optimal energy dissipation to individual nodes. Elmira et.al. [6] proposed a method to recovery of fault node of fault cluster head by apply Genetic Algorithm. Khalilzad, Elmira Moghaddami et.al. [7] To recovery of faulty node depend upon the distance between node to cluster head, energy of cluster head and remaining energy of cluster head. Elmira MK, SanamH et.al. [8] proposed a method to remove cluster head, if faults occurred due to cluster head. Authors [9-14] proposed research based on cluster head recovery algorithms and the cluster head get good benefit.

\section{Proposed Work}

In proposed work introduced Direct Diffusing Algorithm (DDA) and Genetic Algorithm (GA). The Direct Diffusion Algorithm [15] uses interest, exploratory of data, reinforcement and data messages. DDA is implemented for healthiness, improve energy efficiency and reduces data flow on network transmission these all consider for power management issues. DDA is mostly query driven protocol for communication. By communicating method is collected data is match to query from base node then send its appropriate message. The sensing devices are in a network path. The routes are in circular form for broadcasting the message.

By applying Genetic algorithm in literature it is referred as Naturally Inspired Algorithm [16-17], fault node recovery is implemented for increasing of sensor network life time. By processing routing the sensor nodes are lose their energy and become threshold value and become to faulty nodes. In that by using Genetic algorithm applying for fault node recovery of that node. As per this process, reduces cost and also enhance the life time of sensor nodes in clustering approach.

\section{A. Genetic Algorithm}

The proposed Genetic algorithm for fault node recovery in clustering approach is discussed. This process is population (set of solutions) transfer to reproducing (new population) the detection of fault node is purely based on fitness value of nodes.
Step 1: The process not functioning as per expecting results, then replace.

Step 2: Calculate fitness function by replace node information based on expected solution.

Step 3: Based on fitness value the selection is considered.

Step 4: Crossover used for implementation of single crossover process.

Step5: Mutation used to flip nodes selection in randomly and also to reuse the paths for maximize sensor network lifetime.

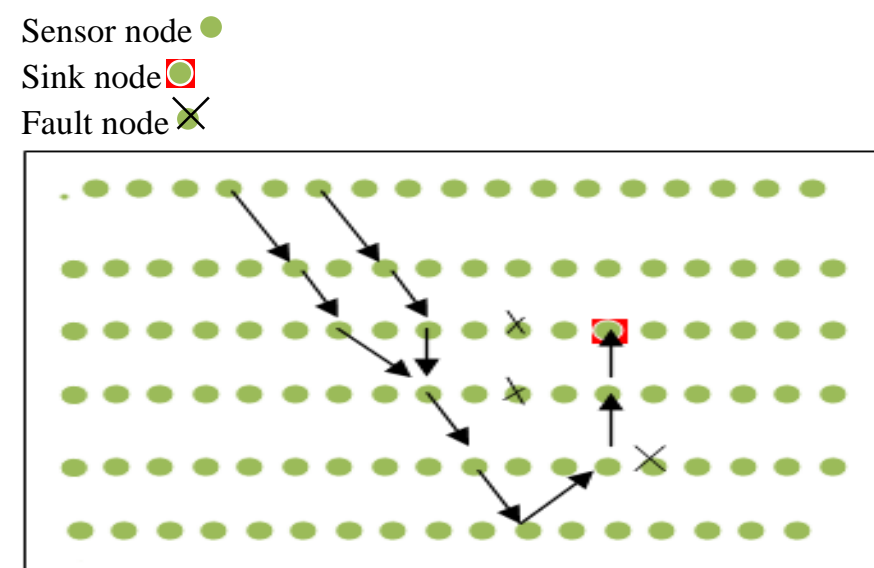

Fig1: Avoiding dead nodes in a clustering network and finding of path by Direct Diffusion algorithm

For implementing GA algorithm following steps are as follows:

i. Initialization - In initialization, chromosomes are generating - the number of chromosomes i.e., genes as per number of sensor nodes in a communication network. Every chromosome initiated by a value generated as fitness value either 0 or 1 .

\begin{tabular}{|c|c|}
\hline Number of Nodes & Fitness value \\
\hline 02 & 01 \\
\hline 04 & 00 \\
\hline 05 & 01 \\
\hline 07 & 00 \\
\hline 09 & 01 \\
\hline 10 & 00 \\
\hline
\end{tabular}

Table 1: Number of nodes and their fitness values

In the above Table number of nodes and their fitness value is represented. Table shows the length of chromosome is 6 . The randomly generate value is either 0 or 1 . The length of chromosome is deactivated nodes in a clustering. The node should replace if the value is 1 .

ii. Evaluation - In this process, fitness value is generated and evaluate as a fitness function. The fitness function used to recovery of fault nodes and reuse to find 
network paths also to replace or change non-functioning of sensor nodes.

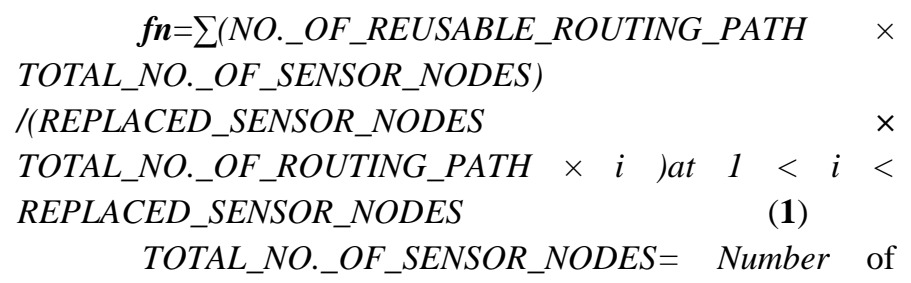

replace /change nodes with grade value at $\mathrm{i}$.

ROUTING_PATH $=$ Number of paths that are reusable from sensor nodes with their grade value at $i$.

TOTAL_NUMBER_OF_SENSOR_NODES = Total

number of sensor nodes in a network.

TOTAL_NUMBER_OF_ROUTING_PATH = Total

number of routing path in a network

iii. Selection- In this process new genes are to replace poorer genes. According to selection of higher fitness value node algorithm eliminate lower fitness value genes. The higher fitness value genes are selected for mating process and remaining worst-case genes should remove.

iv. Crossover - In this process generate new chromosomes. To produce new offspring, select two different chromosomes for mating process. In crossover end, the genes selected in between initial and last genes of parent individual genes. The genes is exchanged and determined, in the process of division of each individual gene on both side of the crossover end.

\section{Performance Evaluation}

The process of simulation for random of 1000 iterations. The model containing the parameters Total Number of deployed in distributed area. The designed for simulation results near about 1000 sensor nodes. The calculations of probability of fault (P), fault_alarm_ratio (FAR) and data accuracy (DA) of fault tolerance in a network, fitness value of Genetic Algorithm (GA). Simulation results contain network, battery, channel and energy models for performance evaluation.

In Network model the area $\mathrm{L} * \mathrm{~B}$ squire meters for deployment of Distributed Sensor Network (DSN). The sensor nodes are randomly deployed in fixed network are for perform Fault_Alarm_Ratio (FAR), Data Accuracy (DA) of present networking. Genetic Algorithm performing by chromosomes lowest fitness value and generate fault status. For processing of media channel access S-MAC protocol applied. The sink node receives all packets with source node information. In that time, the source node is in off state mode.

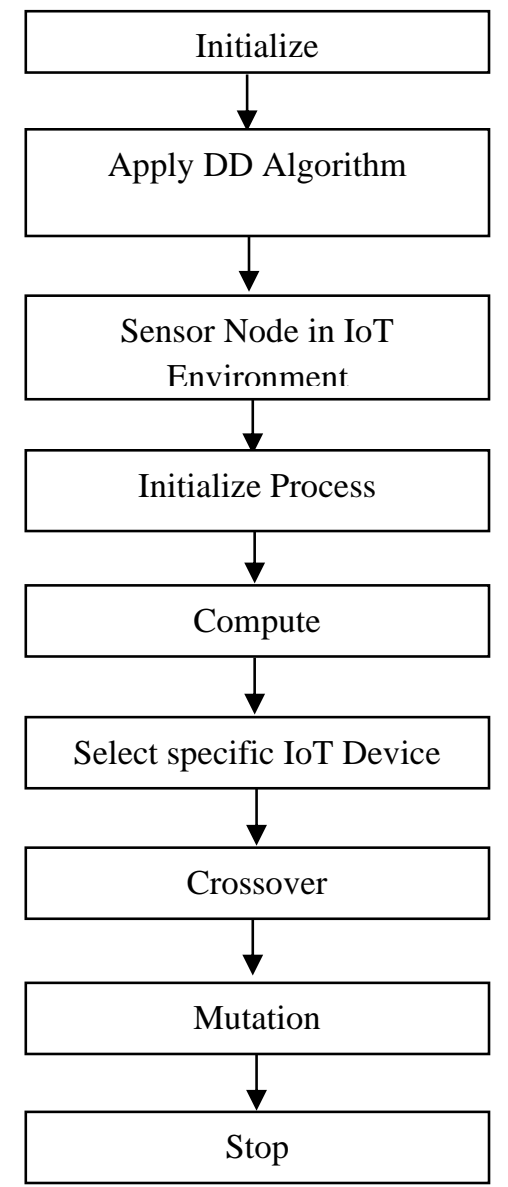

Fig. 2: Flowchart for implementation process GA

In Battery model every sensor is having some battery. The residual energy is remaining energy of battery. The node is randomly located with initial energy. If sensor transmits the packets or sensor receives a data packet from base-station, the node consumes some energy. Energy model in all sensor nodes transmission the radio transmission performing the power control. For reaching fault tolerance status, the node having minimum energy. For a given time, sufficient energy for Energy Transmit (ET) and energy sensing (ES) for k' bit packet to other sensor node for a distance of $\mathrm{d}^{\prime}$ meters is Energy Nodes (EN) for a node. The calculations of total energy as below.

Total Energy $(T E)=($ ENERGY_FOR_SENSING $x$ PACKET_OF_K_BIT $)+($ ENERGY_TRANSMISSION $x$ $\left.P A C K E T \_O F \_K \_B I T\right) * D I S T A N C E \_O F_{-} D_{-}$

In above consider PK as Packets size in terms of bits. The following are parameters are used for different performance metrics in proposed research paper. 


\begin{tabular}{|l|c|l|}
\hline Parameters & Notations & Data \\
\hline Simulation area & L & $1000 \mathrm{~m}$ \\
\hline Width & B & $1000 \mathrm{~m}$ \\
\hline Number of used nodes & NN & 1000 nodes \\
\hline Message transmission range & $\mathrm{CR}$ & $250 \mathrm{~m}$ \\
\hline Type of selection & $\mathrm{Sr}$ & $\begin{array}{l}\text { Selection of wheel } \\
\text { (Roulette) }\end{array}$ \\
\hline Type of crossover & $\mathrm{Cr}$ & Two cross points \\
\hline Crossover possibility & $\mathrm{Cp}$ & $0.07-1.10$ \\
\hline Mutation possibility & $\mathrm{Mp}$ & $0.20-0.40$ \\
\hline Population density & $\mathrm{PS}$ & 1000 \\
\hline Number of repetitions & $\mathrm{IN}$ & 2000 \\
\hline Energy of each nodes & $\mathrm{EN}$ & 2 Joules \\
\hline Hop bandwidth & HW & $1 \mathrm{mbps}$ \\
\hline Threshold energy & THEN & $0.05 \mathrm{~J}$ Joules \\
\hline Threshold link & THoff & $0.287 \mathrm{mbps}$ \\
\hline Energy of each sensor nodes & Es & $50 \mathrm{~nJ} / \mathrm{bit}$ level \\
\hline Used energy for communication & Er & 50 nJ/bit level \\
\hline
\end{tabular}

Table 2: Parameter values

Fault Alarm Ratio (FAR) -False Alarm Ratio (FAR) is ratio of $n$ number of normal nodes areas fault nodes of total number of present normal nodes. In that $\mathrm{N}$ is total number of nodes. High fault probability i.e. high fault alarm rate.

Table 3 - Fault Alarm Ratio (FAR)

\begin{tabular}{|l|l|l|l|}
\hline No. of Rounds & 0 & 50 & 100 \\
\hline NIC Algorithms & GA & DD & EIFDA \\
\hline FAR value & 0.1 & 0.3 & 0.5 \\
\hline
\end{tabular}

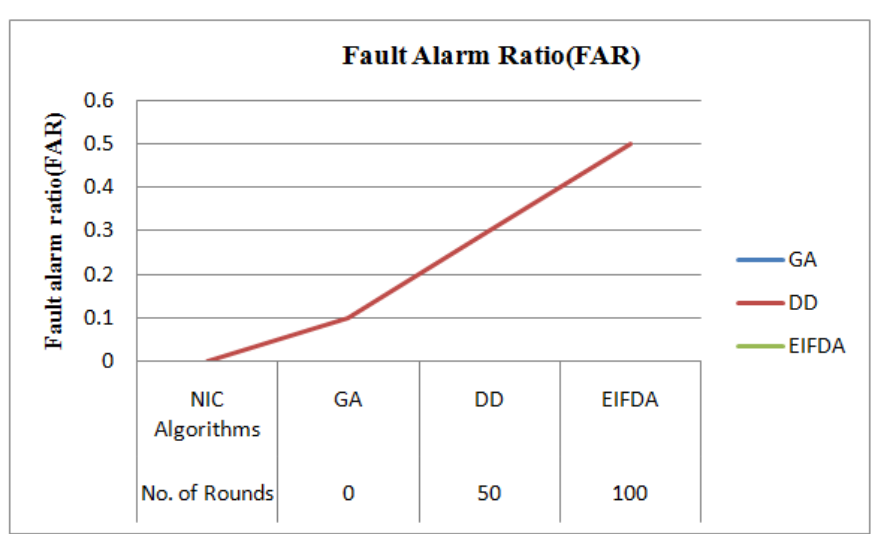

Fig. 3: Fault alarm ratio (FAR) verses total number of sensor nodes (0-150)

Figure 3 shows FAR cannot show high because of less sensor nodes are scattered at distance positions. For that reason fault alarm ratio not high. In DD, while implementing routing from source to destination, at first the faulty sensor nodes are avoided. When comparing with AODV, the fault alarm ratio is lesser in DD. But in Genetic algorithm, by chromosome concept, the fault nodes avoiding and also choose closer active sensor node by implement cross-over method.

Fault probability - The packets are sending from source to destination node without avoiding fault nodes is fault expectation in wireless sensor concept.
Table 4: Probability Ratio (PR)

\begin{tabular}{|l|c|c|c|}
\hline No. of Rounds & 0 & 50 & 100 \\
\hline NIC Algorithms & GA & DD & EIFDA \\
\hline Probability Ratio value & 0.1 & 0.3 & 0.5 \\
\hline
\end{tabular}

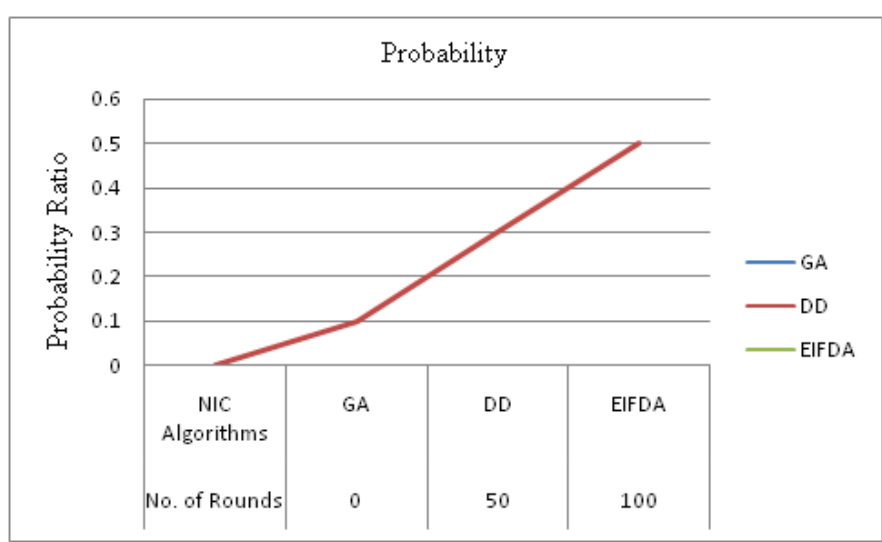

Fig. 4: Fault probability verses total number of sensor nodes (0-to 400)

In Fig. 4, In AODV fault probability is high because without avoiding fault nodes and try to sending packets from source node to destination. In DD, fault probability is less compared with AODV, because DD avoiding fault nodes to sending packets from source sensor node to destination node. But in GA, the fault probability is very less comparing with AODV, DD because, GA by faulty nodes are avoided by implementing chromosome concept and choose active nodes for routing by implementing crossover concept. For that reason in GA fault probability is very less.

Data Accuracy - While sending packets from source to destination by avoiding fault nodes then it is possible to data accuracy.

Table 5: Data Accuracy (DA)

\begin{tabular}{|l|c|c|c|}
\hline No. of Rounds & 0 & 50 & 100 \\
\hline NIC Algorithms & GA & DD & EIFDA \\
\hline FAR value & 0.1 & 0.3 & 0.5 \\
\hline
\end{tabular}

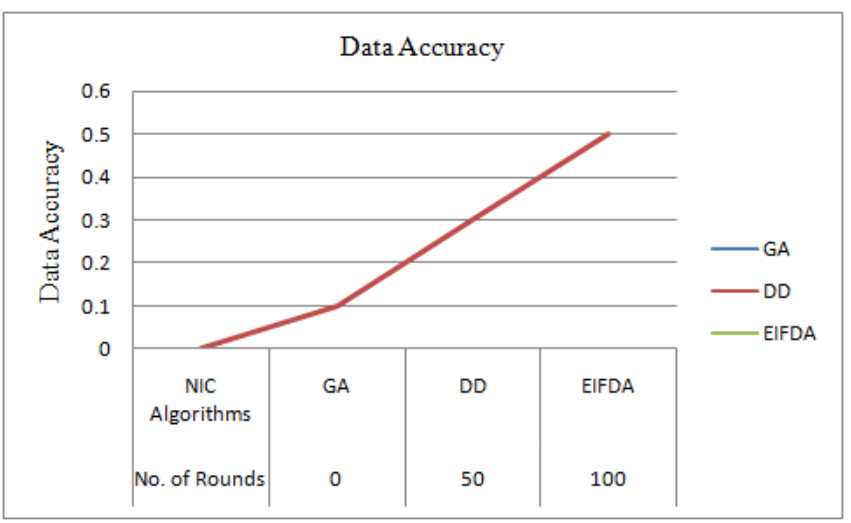

Fig. 5: Data Accuracy (DA) versus total number of sensor nodes (0-150) 
Figure 5 shows In Genetic Algorithm Data Accuracy is higher when comparing with DD and AODV. Because in Genetic Algorithm faulty nodes are detected and avoided. The faulty nodes not detected for that data accuracy in AODV is less. In DD, data accuracy is higher than AODV faculty nodes are detected but not avoid.

\section{Conclusion}

In this paper, efficiency of nature inspired computing technique i.e. Genetic Algorithm to detect fault nodes in a clustering and avoidance for appropriate routing path is described. GA by faulty nodes is avoided by implementing chromosome concept and chooses active nodes for routing by implementing crossover concept. By applying GA the fault tolerance get by fault probability, fault alarm ratio and data accuracy better results when compared with DD as well as AODV. By GA concept of life span of network, network accuracy, efficiency and fault tolerance capability is improved.

\section{References}

[1] Raza HA, Sayeed G, Sajjad H "Selection of cluster heads in wireless sensor networks using bayesian network". In: Proceedings of international conference on computer, electrical, systems, science and engineering, (2010) pp 1-7.

[2] Paradis, Lilia, and Qi Han."A survey of fault management in wireless sensor networks." Journal of Network and systems management 15.2 (2007): 171190.

[3] Cardei, Mihaela, Shuhui Yang, and Jie Wu."Faulttolerant topology control for heterogeneous wireless sensor networks." 2007 IEEE International Conference on Mobile Adhoc and Sensor Systems.IEEE, 2007.

[4] Momani, Mohammad, SubhashChalla, and Rami Alhmouz."Bayesian fusion algorithm for inferring trust in wireless sensor networks." Journal of networks (2010).

[5] Chakraborty, Ayon, Swarup Kumar Mitra, and MrinalKantiNaskar. "A Genetic algorithm inspired routing protocol for wireless sensor networks." International Journal of Computer Intelligence-Theory and Practice 6 (2011).

[6] ElmiraMK,SanamH (2012) "Recovery of faulty cluster head sensor by using genetic algorithm". Int $J$ ComputSci Issues 9(1):141-145.

[7] Khalilzad, Elmira Moghaddami, and SanamHosseini."Recovery of Faulty Cluster Head Sensors by Using Genetic Algorithm (RFGA)." International Journal of Computer Science Issues (IJCSI) 9.4 (2012): 141.

[8] Elmira MK, Sanam H "Recovery of faulty cluster head sensor by using genetic algorithm". Int J ComputSci Issues 9(1)(2012):141-145.

[9] Lokesh BB, Nalini N "Energy aware based fault tolerance approach for topology control in distributed sensor networks". Int J High Speed Netw 18(3) (2012):197-210.

[10] Alaa FO, Mohammed Al "Improving the performance of the networks using genetic algorithm". In: Proceedings of international conference of advances in computer networks and its security, vol 2, no 3, pp 117-120, 2012.

[11] Myeong HL, Yoon HC "Fault detection of wireless sensor networks". JComputCommun31:3469-3475, 2008.

[12] Xiaofeng H, Xiang C, Lloyd LE, Chien-Chug S "Faulttolerant relay node placement in heterogeneous wireless sensor networks". IEEE Trans Mob Comput 9(5)(2010):643-656.

[13] Biao C, Ruixiang J, Kasetkasem T, Varshney PK "Channel aware decision fusion in wireless sensor networks". IEEE Trans Signal Process 52(12)2004:3454-3458.

[14] Darrell W (1994) "A genetic algorithm tutorial". J Stat Comput 4:65-85.

[15] Iyengar SS, Ankit T, Brooks RR (2004) An overview of distributed sensors network. Chapman and Hall/CRC, London, pp 3-10. http://books.google.com/books/about/ Distributed-sensor-networks. html?id=Nff5.

[16] Dixit, Manish, Nikita Upadhyay, and Sanjay Silakari. "An exhaustive survey on nature inspired optimization algorithms." International Journal of Software Engineering and Its Applications 9, no. 4 (2015): 91104.

[17] Singh, Abhilash, Sandeep Sharma, and Jitendra Singh. "Nature-inspired algorithms for Wireless Sensor Networks: A comprehensive survey." Computer Science Review 39 (2021): 100342. 